\title{
AVALIAÇÃO DA INTENSIDADE DE DOR PÓS-OPERATÓRIA EM PACIENTES SUBMETIDOS A PROCEDIMENTOS CIRÚRGICOS PERIODONTAIS - CORRELAÇÃO ENTRE DIFERENTES ESCALAS
}

\author{
EVALUATION OF POSTOPERATIVE PAIN INTENSITY IN \\ PATIENTS WHO UNDERWENT PERIODONTAL SURGERY - \\ A CORRELATION BETWEEN DIFFERENT SCALES
}

\author{
Rodrigo Cavassim ${ }^{1 *}$, Audilene Karine Bianchi ${ }^{1}$, Claudinéia Wendler Tozetto ${ }^{1}$, \\ Gibson Luiz Pilatti' ${ }^{2}$ Fábio André dos Santos ${ }^{2}$ \\ 1* Autor para contato: Universidade Estadual de Ponta Grossa - UEPG, Campus em \\ Uvaranas, PIBIC/CNPq/UEPG, Ponta Grossa, PR, Brasil; (42) 446-1303; \\ e-mail: rcavassim@hotmail.com \\ 1 Universidade Estadual de Ponta Grossa - UEPG, Campus em Uvaranas, \\ Departamento de Odontologia, Ponta Grossa, PR. \\ 2 Universidade Estadual de Ponta Grossa, Campus em Uvaranas, Departamento de \\ Odontologia e Curso de Especialização em Periodontia da EAP-ABO/Ponta Grossa, \\ PR, Brasil.
}

Recebido para publicação em 21/11/2003

Aceito para publicação em 23/12/2003

\begin{abstract}
RESUMO
Dor pós-operatória é freqüentemente relatada por pacientes submetidos a cirurgia periodontal. Como a dor é de natureza subjetiva, necessitamos de instrumentos que nos auxiliem a avaliar a sua intensidade para estabelecermos a terapêutica adequada. Este estudo comparou 3 diferentes escalas destinadas a avaliação de dor. Foram utilizadas a escala visual analógica (VAS), a escala numérica de 101 pontos e a escala verbal de 4 pontos. As escalas foram aplicadas a pacientes submetidos a tratamento periodontal cirúrgico, que consistiu de um retalho total para descontaminação da superfície radicular. Os pacientes receberam instruções sobre as escalas, bem como um prontuário para avaliação de dor. Os dados coletados foram tabulados e submetidos a análise estatística pelo teste de correlação de Spearman, adotando como nível de significância o valor de p $\leq 0,05$. Os resultados indicaram haver uma correlação positiva estatisticamente significante entre a VAS e a escala numérica de 101 pontos, entre a VAS e a escala verbal de 4 pontos e também entre a escala numérica de 101-pontos e a escala verbal de 4 pontos. Pôde-se concluir que as metodologias mostraram-se efetivas para avaliação da intensidade de dor pós-operatória em procedimentos cirúrgicos periodontais.
\end{abstract}


Palavras-chave: cirurgia periodontal, dor pós-operatória, escalas para avaliação de dor

\begin{abstract}
Postoperative pain is frequently related by patients submitted to periodontal surgery. Because pain is a subjective variable, it becomes necessary to apply scales which facilitate pain intensity determination. This study compared three different scales for pain assessment: 1) visual analogue scale (VAS); 2) 101-point numerical rating scale (NRS-101); 3) Four-point verbal rating scale (VRS-4). The scales were applied to patients who underwent scaling and root planing through mucoperiostal periodontal flap surgery. The patients received an initial explanation on how to assess pain intensity on a registration sheet every hour for an 8-hour period and three times a day for the following three days. The data were analyzed using Spearman's correlation test, with $\mathrm{p} \leq 0.05$. The results showed that there was a positive correlation between VAS and NRS-101, between VAS and VRS4 and between NRS-101 and VRS-4 scales. Therefore, all the three scales showed to be effective for pain assessment after periodontal surgery.
\end{abstract}

Key words: periodontal surgery, postoperative pain, scales for pain assessment

\section{Introdução}

A dor normalmente é associada a dano tecidual e possui uma natureza extremamente subjetiva (Cardoso Jr. e Camparis, 2002). A subjetividade da dor acaba refletindo-se na dificuldade que temos quando buscamos um método para sua avaliação. Na odontologia, a dor pode estar presente em dois momentos: quando associada a uma patologia que acomete a cavidade bucal ou como resultado de uma intervenção cirúrgica. Dos procedimentos odontológicos, os que apresentam maior expectativa de dor pós-operatória são as exodontias - em especial de terceiros molares - e os procedimentos cirúrgicos periodontais (Cooper et al, 1976; Gallardo e Rossi, 1990; Gallardo e Rossi, 1992; Vogel et al, 1992; Matthews e McCuloch, 1993; Trombelli et al, 1996). Há a necessidade do estabelecimento de um protocolo para controle de dor pósoperatória em cirurgias periodontais, porém, primeiramente é necessário avaliar as metodologias já propostas para medir a intensidade da dor decorrente desses procedimentos, a fim de permitir a comparação entre diferentes protocolos medicamentosos que visem controlar a dor pós-operatória, o que motivou a realização deste estudo.

\section{Revisão da literatura}

Diferentes metodologias vêm sendo utilizadas para avaliação da dor pós-operatória em odontologia, bem como na tentativa de verificar a eficácia de diferentes protocolos terapêuticos para controle de dor.

Com o objetivo de testar a eficácia analgésica do flurbiprofeno (anti-inflamatório não-esteroidal AINE) foi realizado um estudo duplo cego, comparando-o com o acetaminofeno e placebo no controle da dor após tratamento periodontal cirúrgico. Participaram deste estudo 63 pacientes adultos que necessitavam de tratamento periodontal cirúrgico. A cirurgia consistiu de retalho total seguido de raspagem e alisamento radicular em um segmento de seis dentes. Para avaliar a intensidade de dor foi utilizada a escala verbal de 4 pontos, a qual consiste de 4 adjetivos, sendo que o paciente deveria escolher uma das alternativas disponíveis que melhor representasse a dor que estivesse sentindo $(0=$ ausência de dor, $1=$ dor leve, 2 = dor moderada, 3 = dor severa). A escala foi aplicada em intervalos de 1 hora entre cada avaliação (iniciando 3 horas após a utilização do primeiro comprimido). A escala mostrou-se adequada na avaliação da intensidade de dor pós-operatória, apresentando curvas de 
tempo que mostraram que o flurbiprofeno apresentou melhor eficácia que o placebo em todos os tempos avaliados; o acetaminofeno foi superior ao placebo somente na segunda hora de avaliação; o flurbiprofeno foi superior ao acetaminofeno em todos os tempos de avaliação (Gallardo e Rossi, 1990).

O controle da dor durante procedimentos operatórios é importante em odontologia, proporcionando maior conforto ao paciente. O método mais comum para controle da dor é a utilização de anestesia local. Carr e Horton (2001) realizaram uma avaliação clínica entre 2 tipos de anestésicos tópicos utilizados para controle da dor causada pelas injeções convencionais de anestésico e raspagem e alisamento radiculares. Foram atendidos 40 pacientes com idade entre $21 \mathrm{e}$ 70 anos, que apresentavam bolsas periodontais com profundidade de 4 a $6 \mathrm{~mm}$ e sangramento à sondagem em pelo menos 2 pré-molares de cada quadrante. Foi avaliada a efetividade de um sistema de liberação de lidocaína (lidocaine transoral delivery system - LDS), e do gel de benzocaína. Após a aplicação das substâncias, testou-se o poder de anestesia das mesmas, através de uma simulação de injeção, seguida de instrumentação subgengival. Para avaliar a dor foram utilizadas a escala verbal de 5 pontos (5-point Verbal Rating Scale - VRS-5) e a escala visual analógica (VAS). A VRS- 5 consiste de 5 adjetivos e a cada um é atribuído um valor numérico ( $0=$ sem dor; $1=$ dor leve; 2=dor moderada; $3=$ dor forte; $4=$ dor muito forte), sendo que o paciente deveria marcar um deles no momento da avaliação da dor. Os resultados mostraram que o LDS foi mais efetivo que o BGP na anestesia tópica e também para raspagem e alisamento radicular em ambos os arcos (Carr e Horton, 2001).

Um estudo recente testou a eficácia de um gel anestésico (lidocaína $25 \mathrm{mg} / \mathrm{g}$ associada a prilocaína $25,5 \mathrm{mg} / \mathrm{g}$ ) utilizado para controle da dor em procedimentos de raspagem e alisamento radicular. Para avaliar a eficácia do gel, foram utilizadas a escala visual analógica (VAS) e também a escala verbal de 5 pontos (VRS-5). A VAS demonstrou uma redução significativa da dor para o gel anestésico quando comparado ao placebo $(p<0,0005)$. A VRS-5 mostrou que $90 \%$ dos pacientes tratados com o gel anestésico relataram ausência de dor ou dor leve comparando com os $64 \%$ tratados com o placebo $(p<0,001$ ) (Jeffcoat et al, 2001).
Num estudo realizado com o objetivo de avaliar e quantificar as diferenças de percepção dos pacientes entre os tratamentos cirúrgico e não cirúrgico, também para testar a sensibilidade do método de avaliação, foi aplicado um questionário à 162 pacientes. Os dados avaliados para cada paciente incluíram o desconforto sentido durante o tratamento periodontal, dor pós-operatória, sensibilidade e edema, complicações funcionais (alimentação e fala) e comparação do desconforto do tratamento periodontal em relação a um procedimento restaurador ou preparo coronário. Para fazer a avaliação desses dados foi utilizada uma escala visual analógica (VAS) aplicada ao paciente em cada visita à clínica. A escala visual analógica (VAS) reproduziu as sensações sentidas pelos pacientes. Também se mostrou um método simples de aplicar, confiável e válido (Matthews e McCulloch, 1993).

Para a avaliação da intensidade de dor após raspagem e alisamento radicular (RAR), Pihlstrom et al (1999) utilizaram a escala de dor de Heft-Parker, que consiste de uma linha horizontal de 170mm cujos extremos indicam ausência de dor e dor máxima possível. Cada paciente deveria marcar com um traço vertical o ponto que melhor indicasse a dor que estava sentindo no momento da avaliação. A média do início da dor foi de 2,8 horas após o término da RAR, com duração média de 6,1 horas. $23 \%$ dos pacientes utilizaram analgésicos para aliviar a dor. Os pacientes que participaram do estudo tiveram dor com duração e intensidade significantes após RAR, com pico entre 2 e 8 horas após a RAR. Devido a essas razões deveria ser considerada a utilização de analgésicos que tivessem pico de ação de 2 a 8 horas para aliviar a dor após a realização da raspagem e alisamento radicular

Diferentes situações nas quais há expectativa de dor têm sido utilizadas para se avaliar a precisão das escalas de medição de dor. Huskisson (1974) discutiu a utilização da escala de descrição simples, a escala visual analógica e também métodos não-verbais como a observação da expressão facial. Ele observou que a escala visual analógica mostrou-se mais sensível que a escala de descrição simples na avaliação da dor. Para o autor, mais importante que avaliar a intensidade da dor seria a avaliação do alívio desta. Para isso ele utilizou a escala de descrição simples, a qual mostrou-se adequada para tal situação. Também observou que o alívio da dor leva a uma diminuição de catecolaminas 
excretadas na urina, sendo esse um importante método indireto para avaliar o alívio da dor.

Scott e Huskisson (1976) testaram seis diferentes tipos de escalas visuais analógicas e escalas gráficas em 100 pacientes de ambulatório que sentiam dor. Somente duas escalas deram distribuição uniforme de resultados e foram consideradas satisfatórias: a escala visual analógica e a escala gráfica de dor. Ambas se mostraram mais sensíveis que a escala descritiva simples.

Jensen et al (1986) apresenta seis diferentes escalas para avaliar a intensidade de dor: escala analógica visual (visual analogue scale - VAS); escala numérica de 101 pontos (101-point numerical rating scale - NRS-101); escala em quadrados de 11 pontos (11 point Box scale - BS-11); escala comportamental de 6 pontos (6-point behavioral rating scale - BRS-6); escala verbal de 4 pontos (4-point verbal rating scale - VRS-4); escala verbal de 5 pontos (5-point verbal rating scale - VRS-5). Diversos fatores analisados demonstraram que as seis escalas estudadas são instrumentos úteis e confiáveis para avaliação de dor. Porém, a NRS-101 mostrou-se extremamente simples de ser empregada, tendo como principal vantagem o fato de poder ser utilizada nas formas escrita ou verbal, ao contrário da VAS, a qual pode ser utilizada apenas na forma escrita/gráfica.

A escala visual analógica(VAS) éuminstrumento útil que pode ser aplicado para avaliar a dor. Por ser simples e de fácil aplicação ela pode ser utilizada na odontologia para a avaliação de dor após procedimentos de raspagem e alisamento radicular. Deste estudo participaram 150 pacientes (90 mulheres e 60 homens com idade média de 54,5 anos) submetidos à terapia periodontal, incluindo cirurgia. Para avaliação do desconforto pós-operatório foi utilizada a VAS. Os pacientes tinham opção de utilizar, caso julgassem necessário, uma associação de paracetamol e codeína. Os procedimentos avaliados foram: tomadas radiográficas, exame periodontal, raspagem e alisamento radicular, anestesia (região inferior), cirurgia (região inferior), anestesia (região anterior superior), cirurgia (região superior anterior). A média dos valores obtidos para a intensidade de dor foi baixa para todos os procedimentos avaliados. O escore médio maior foi obtido na anestesia da região superior anterior. Pequenas diferenças foram encontradas quando comparados os ní- veis de desconforto relatados por homens e mulheres. Foram observados níveis de desconforto muito baixos associados a procedimentos cirúrgicos e não-cirúrgicos (Fardal et al, 2002).

\section{Material e métodos}

Tomaram parte deste estudo cego e randomizado, um total de 10 pacientes de ambos os sexos, entre 25 e 55 anos. Cada paciente foi submetido a cirurgia periodontal em 3 quadrantes. Para cada procedimento cirúrgico foram realizadas 17 avaliações da intensidade de dor em tempos diferentes após a cirurgia. Dessa forma, foram geradas 510 avaliações de intensidade de dor. Para inclusão no estudo, os paciente deveriam ser portadores de periodontite crônica generalizada, tendo sido submetidos, previamente, a tratamento periodontal básico, o qual envolveu orientação sobre higiene bucal, controle de placa e raspagem e alisamento radicular na Clínica de Periodontia da Universidade Estadual de Ponta Grossa. Após reavaliação, os pacientes que ainda apresentassem sinais clínicos de atividade inflamatória, como presença de sangramento à sondagem e/ou supuração em pelo menos três quadrantes foram encaminhados ao Curso de Especialização em Periodontia da Escola de Aperfeiçoamento Profissional da Associação Brasileira de Odontologia - Regional de Ponta Grossa (EAP-ABO/ PG) para submeterem-se a tratamento periodontal cirúrgico em pelo menos três quadrantes.

Os critérios de exclusão adotados foram: a) gestantes; b) pacientes de risco à endocardite bacteriana; c) diabéticos descontrolados; d) pacientes portadores de gastrite, úlcera gástrica ou duodenal; e) pacientes hipertensos descontrolados; f) pacientes que já estivessem fazendo uso recentemente de drogas analgésicas e antiinflamatórias.

Os pacientes foram esclarecidos da necessidade de complementação cirúrgica nos sítios portadores de atividade inflamatória e assinaram um termo de consentimento pós-informação, ficando livres para abandonarem a pesquisa a qualquer momento, sem prejuízo ao tratamento ao qual estavam sendo submetidos.

Foram registrados em ficha apropriada os da- 
dos pessoais, anamnese e exame clínico periodontal dos pacientes selecionados para o estudo. Cada quadrante foi submetido - com um intervalo mínimo de 30 dias entre cada procedimento - à cirurgia periodontal que consistiu na realização de retalho total de acesso para raspagem e alisamento radicular com instrumentos manuais e ultra-sônicos. As intervenções foram realizadas sob anestesia local, tendo a duração de cada procedimento sido anotada em ficha própria. Empregando-se um modelo de boca dividida, foi determinado aleatoriamente para cada quadrante, um dos seguintes protocolos de medicação pré-operatória para prevenção da dor, segundo dose habitual preconizada pelos fabricantes: a) grupo placebo: 01 comprimido placebo 1 hora antes da cirurgia, seguido de outro comprimido placebo 8 horas depois; b) grupo AIE: 01 comprimido de $4 \mathrm{mg}$ de dexametasona (Decadrom ${ }^{\circledR}$ ) $(0,06 \mathrm{mg} / \mathrm{Kg}) 1$ hora antes da cirurgia, seguido de outro comprimido 8 horas depois; c) grupo AINE: 01 cápsula de 200mg de Celecoxib (Celebra $\left.{ }^{\circledR}\right)$ (3mg/Kg) 1 hora antes da cirurgia, seguido de outra cápsula 12 horas depois. Para todos os grupos experimentais foi fornecido aos pacientes medicação analgésica de suporte (12 comprimidos de paracetamol 750mg), a qual poderia ser utilizada pelo paciente quando necessário, em intervalos mínimos de 06 horas.

A intensidade de dor pós-operatória foi determinada pelos pacientes, fazendo-se o registro em ficha apropriada. Para tanto, cada paciente recebeu um prontuário para avaliação de dor constituído de 17 fichas; cada ficha contendo as três escalas (figura 1). Cada paciente preencheu a ficha de avaliação no horário prédeterminado, sendo que a primeira avaliação foi 1 hora após o término da cirurgia (após terem sido realizadas as suturas), prosseguindo a cada hora até a $8^{\text {a }}$ hora após o fim do procedimento. Nos 3 dias que se seguiram ao dia do procedimento, a avaliação foi feita por três vezes ao dia (pela manhã, no horário do almoço e à noite).

PRONTUÁRIO PARA AVALIAÇÃo DE DOR

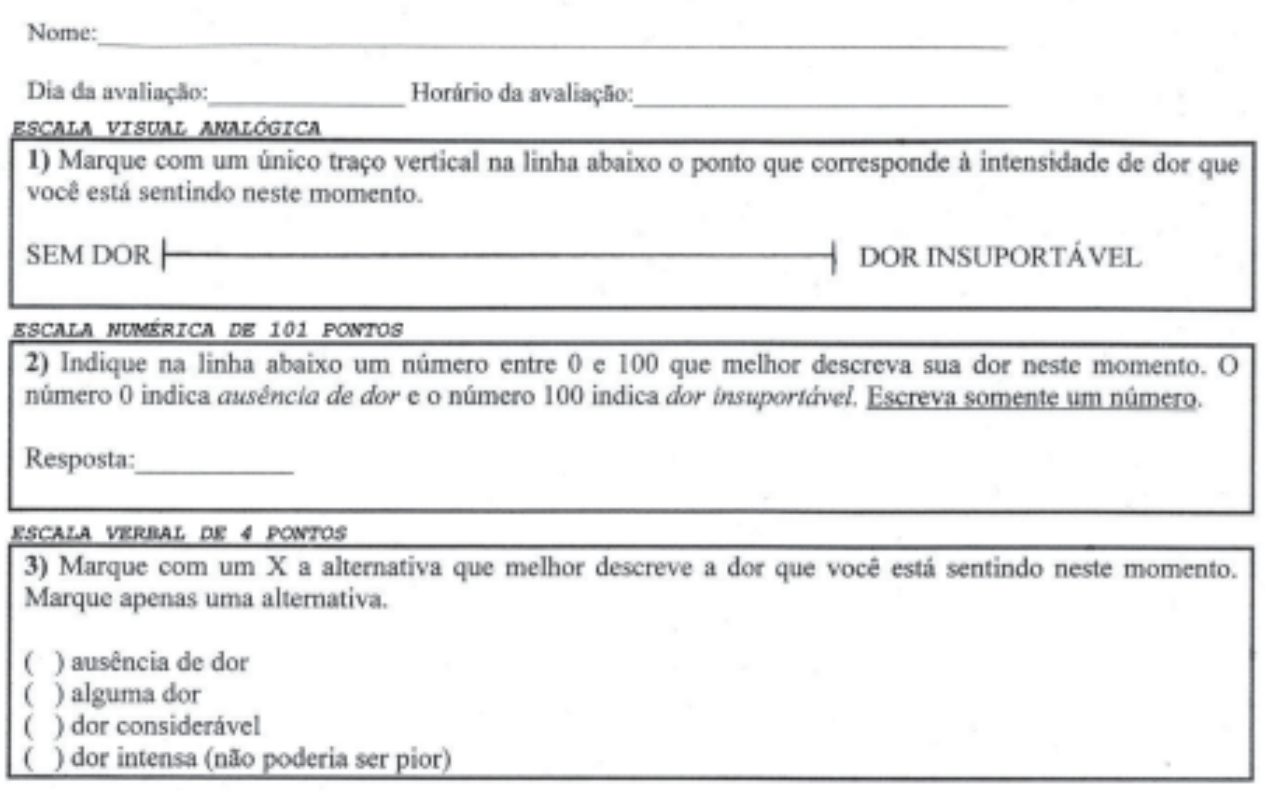

Figura 1 - Ficha utilizada no prontuário de avaliação da intensidade de dor.

As três diferentes escalas utilizadas para avaliação da intensidade de dor foram: a) escala analógica visual (VAS); b) escala numérica de 101 pontos (NRS101); c) escala verbal de 4 pontos (VRS-4) (Jensen et al., 1986). As instruções sobre o preenchimento do prontuário de avaliação de dor foram dadas por 1 pesquisador treinado e repetidas após cada procedimento, constando de informações que auxiliassem ao paciente no preenchimento das escalas para avaliação da dor. A escala visual analógica consiste em uma linha de 
$10 \mathrm{~cm}$ com os dois extremos fechados. Um dos extremos apresentava a indicação "SEM DOR" e no outro “DOR INSUPORTÁVEL”. Cada paciente foi orientado a marcar com um único traço vertical o ponto que melhor correspondesse à intensidade de dor no momento da avaliação. A determinação da intensidade da dor foi feita medindo-se a distância - em milímetros - entre o ponto "SEM DOR” até a marca feita pelo paciente. Na escala numérica de 101 pontos, pediu-se ao paciente que escolhesse um número entre 0 e 100 que melhor representasse a sua dor, sendo que 0 indica “AUSÊNCIA DE DOR” e 100 indica "DOR INSUPORTÁVEL”. O número marcado corresponde à intensidade de dor sentida pelo paciente no momento da avaliação. A escala verbal de 4 pontos consiste de uma lista de adjetivos que descrevem os diferentes níveis de dor: 0 - “ausência de dor”, 1 - “alguma dor”, 2 - “dor considerável”, 3 - “dor intensa (não poderia ser pior)”. A intensidade da dor foi avaliada por cada paciente escolhendo apenas uma das alternativas. Eles foram orientados a escolher, e marcar com um “ $X$ ”, a alternativa mais representativa da dor experimentada no momento da avaliação. Quando os pacientes retornaram após 7 dias para remoção das suturas e controle pós-operatório foi anotada a quantidade de analgésicos utilizados como medicação de suporte. Os dados coletados foram tabulados e submetidos à análise estatística. Foi utilizado o teste de correlação de Spearman, adotando como nível de significância o valor de $p \leq 0,05$.

\section{Resultados}

Os dados obtidos para a VAS e para a NRS101 estão representados na figura 2. O emprego do teste de correlação de Spearman demonstrou uma correlação positiva $(r=0,58)$ estatisticamente significante $(p=0,0001)$ entre as escalas avaliadas. Isso indica que quando há um aumento nos valores de dor na VAS, há também uma tendência de aumento nos valores de dor da NRS-101.

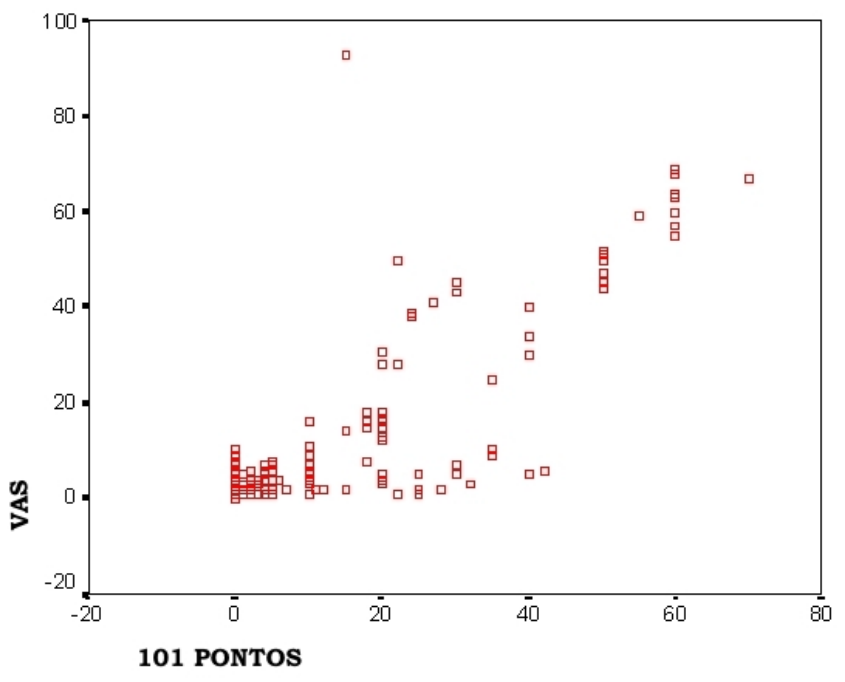

Figura 2 - Correlação entre as escalas VAS e NRS-101 $(r=0,58 ; p=0,0001)$.

A comparação dos dados entre a VAS e a VRS4 apresentados na figura 3 expressa uma correlação positiva $(r=0,579)$ estatisticamente significante $(p=0,0001)$. Essa correlação positiva encontrada indica haver uma tendência de que quando ocorre um aumento nos valores de dor para a VAS, o paciente também relate maiores valores para dor na VRS-4.

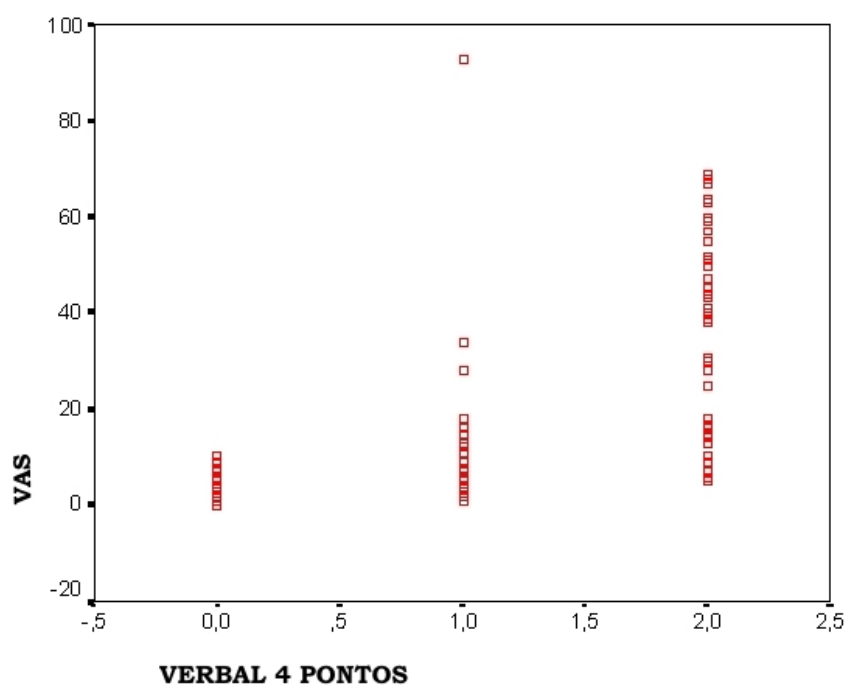

Figura 3 - Correlação entre as escalas VAS e VRS-4 $(r=0,579 ; p=0,0001)$ 
Quando foram comparadas as escalas NRS-101 e VRS-4 (figura 4), observou-se uma correlação fortemente positiva $(r=0,987)$ entre as escalas analisadas. Há uma forte tendência, estatisticamente significante $(p=0,0001)$, de que à medida que os valores de dor aumentem na NRS-101, também aumente a intensidade de dor registrada pelo paciente na VRS4.

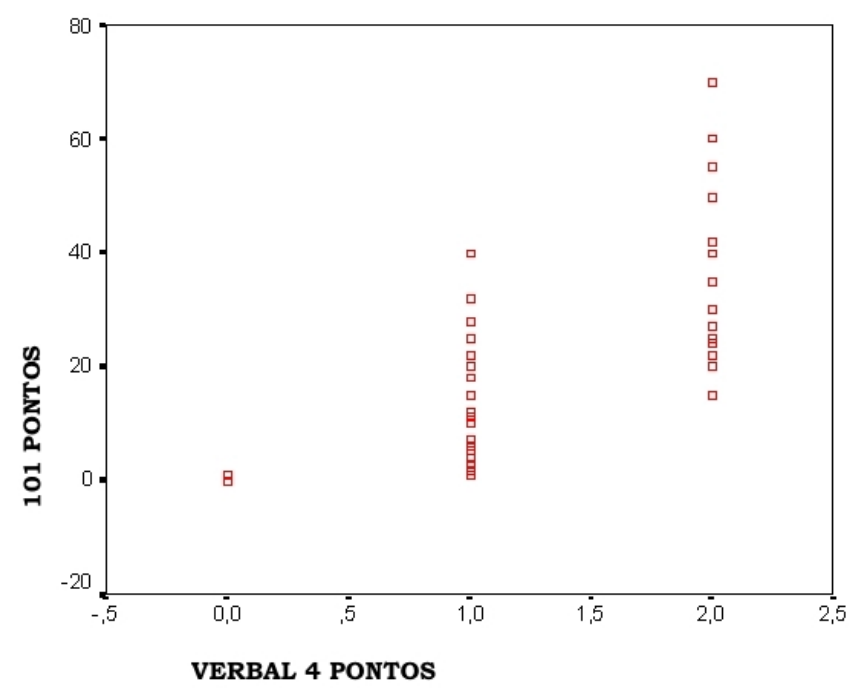

Figura 4 - Correlação entre as escalas NRS-101 e VRS-4 $(r=0,987 ; p=0,0001)$.

\section{Discussão}

A percepção da dor é subjetiva e dependente da percepção individual, havendo uma tendência - não significante estatisticamente - de que mulheres apresentem uma maior intensidade de dor em procedimentos periodontais não cirúrgicos (Pihlstromet al, 1999).

Estudos distinguem dois tipos diferentes de dor: induzida e patológica. A dor induzida ocorre a partir da existência de um estímulo e pode ser expressa verbalmente ou não verbalmente (Houde, 1982). Uma maneira não verbal de expressar a dor seria através da escala visual analógica (visual analogue scale - VAS), na qual a dor é expressa através de uma marca feita na linha de $10 \mathrm{~cm}$ da escala. Outra maneira seria a escala numérica de 101 pontos (esta escala pode ser utilizada tanto na forma verbal como não-verbal), na qual a dor é expressa através da escolha de um número entre $0 \mathrm{e}$ 100. Por outro lado temos as escalas verbais, como a escala verbal de 4 pontos, nas quais a dor é expressa pela escolha de um dos adjetivos apresentados que mais se aproxima da dor experimentada (Jensen et al, 1986). Huskisson (1974) também considera os sinais faciais como sendo demonstração não-verbal de dor. Entretanto, este tipo de fenômeno comportamental não pode ser tido como indicador da severidade de dor.

Diferentes trabalhos foram realizados na busca de metodologias adequadas para avaliação de dor pósoperatória em odontologia (Huskisson, 1974; Scott e Huskisson, 1976; Seymour et al, 1983; Jensen et al, 1986). Os modelos experimentais normalmente utilizados são exodontias de $3^{\circ}$ s molares e cirurgias periodontais, por apresentarem uma maior expectativa de dor pós-operatória (Cooper et al, 1976; Gallardo e Rossi, 1990; Gallardo e Rossi, 1992; Vogel et al, 1992; Mattews e McCulloch, 1993; Trombelli et al, 1996).

Em 1976 Scott e Huskisson realizaram um estudo comparando seis diferentes tipos de escalas visuais analógicas em relação à escala de descrição simples. Foi observada uma correlação fortemente positiva estatisticamente significante entre as diferentes escalas. Em nosso estudo a correlação também foi positiva estatisticamente significante entre as escalas, quando comparadas duas a duas.

Das seis escalas para avaliação de dor propostas por Jensen et al (1986), utilizamos três delas, sendo duas não-verbais (VAS e escala numérica de 101 pontos) e uma verbal (escala verbal de 4 pontos). As três metodologias estudadas mostraram-se adequadas para a avaliação da intensidade de dor pós-operatória em cirurgias periodontais. Observou-se uma correlação positiva $(r=0,58)$, estatisticamente significante $(p=0,0001)$ entre a VAS e a escala numérica de 101 pontos e também uma correlação positiva $(r=0,579)$, estatisticamente significante $(\mathrm{p}=0,0001)$ entre a VAS e a escala verbal de 4 pontos. A VAS já havia sido comparada com as partes três e quatro do McGill Pain Questionaires (MPQ) e apresentou uma correlação positiva $(r=0,69)$ estatisticamente significante $(p<0,001)$ quando comparada às outras categorias do MPQ (Seymour et al, 1983).

Uma correlação fortemente positiva $(r=0,987)$, estatisticamente significante $(p=0,0001)$ foi encontrada quando comparamos a escala numérica de 101 pontos com a escala verbal de 4 pontos. Resultados 
obtidos por Jensen et al, 1986, quando compararam 6 diferentes escalas para avaliação de dor, demonstram que a escala numérica de 101 pontos tem vantagens sobre as outras por ser bastante simples de administrar e também por poder ser aplicada na forma verbal ou não-verbal.

Analisando os resultados obtidos em nosso estudo encontramos uma concordância com o que Mattews e McCulloch (1993) disseram a respeito da VAS. Esta escala conseguiu reproduzir as sensações sentidas pelos pacientes, mostrando-se um método simples de aplicar, confiável e válido. Também concordamos com Jensen et al (1986) sobre a facilidade e praticidade de uso da escala numérica de 101 pontos, já que esta pode ser administrada tanto de maneira verbal quanto escrita e é de fácil compreensão pelo paciente.

\section{Conclusão}

Através dos dados obtidos pôde-se observar que, apesar do caráter subjetivo da dor, houve uma correlação positiva, estatisticamente significante entre as escalas utilizadas, sendo que a correlação foi mais forte entre a escala numérica de 101 pontos e a escala verbal de 4 pontos $(r=0,987)$ do que com as outras escalas utilizadas na avaliação da dor pós-operatória em cirurgias periodontais.

Desta forma, as metodologias estudas mostraram-se adequadas para avaliação da intensidade de dor pós-operatória em procedimentos cirúrgicos periodontais e, que quando empregadas corretamente, são instrumentos úteis nas pesquisas que buscam avaliar protocolos para controle da dor pós-operatória em odontologia.

\section{REFERÊNCIAS}

1 CARDOSO JR., C; CAMPARIS, C. M.A psicologia da doraspectos de interesse do cirurgião dentista. Disponível em: <http://www.odontologia.com.br> Acesso em:31 jan. 2004.

2 CARR, M. P.; HORTON, J. E. Clinical evaluation and comparison of 2 topical anesthetics for pain cause by needle sticks and scaling and root planing. J Periodontol, v.72, n. 4, p. 479-484, Apr 2001.
3 COOPER, S. A.; BEAVER, W. T. A model to evaluate mild analgesics in oral surgery outpatients. Clinical Pharmacology and Therapeutics, v.20, n.2, p.241-250, Aug 1976.

4FARDAL, Ø.; JOHANNESSEN, A. C.; LINDEN, G. J. Patient perceptions of periodontal therapy completed in a periodontal pratice. J Periodontol, v.73, n.9, p.1060-1066, Sept 2002.

5 GALLARDO, F.; ROSSI, E. Analgesic efficacy of flurbiprofen as compared to acetaminophen and placebo after periodontal surgery. J Periodontol, v.61, n.4, p.224-227, Apr 1990.

6 GALLARDO, F.; ROSSI, E. Effects of sodium meclofenamate on postoperative pain following periodontal surgery. J Periodontol, v.63, n.3, p.116-168, Mar 1992.

7 HUSKISSON, E. C. Measurement of pain. Lancet, v.9, n.2, p.1127-1131, Nov 1974.

8 HOUDE, R. W. Methods for measuring clinical pain in humans. Acta anaesth Scand, v.74 (suppl.), p.25-29, 1982.

9 JEFFCOAT, M. K.; GEURS, N. C.; MAGNUSSON, I.; MACNEILL, S. R.; MICKELS, N.; ROBERTS, F.; ROBINSON, P. SALAMATI, A.; YUKNA, R. Intrapocket anesthesia for scaling and root planing: results of a double-blind multicenter trial using lidocaine prilocaine dental gel. J Periodontol, v.72, n. 7, p.895-900, Jul 2001.

10 JENSEN, M. P.; KAROLY, P.; BRAVER, S. The measurement of clinical pain intensity: a comparison of six methods. Pain, v.27, n.1 p.117-126, Oct 1986.

11 MATTHEWS, D. C.; MCCULLOCH, C. A. G. Evaluating patient perceptions as short-term outcomes of periodontal treatment: a comparison of surgical and non-surgical therapy. J Periodontol, v.64, n.10, p.990-997, Oct 1993.

12 PIHLSTROM, B. L.; HARGREAVES, K. M.; BOUWSMA, O. J.; MYERS, W. R.; GOODALE, M. B.; DOYLE, M. J. Pain after periodontal scaling and root planing. J Am Dental Assoc, v.130, n.6, p.801-807, Jun 1999.

13 SCOTT, J.; HUSKISSON, E. C. Graphic representation of pain. Pain, v.2, n.2, p.175-184, Jun 1976.

14 SEYMOUR, R. A.; CHARLTON, J. E.; PHILLIPS, M. E. An evaluation of dental pain using visual analogue scales and the McGill pain questionnaire. J Oral Maxillofac Surg, v.41, n.10, p.643-648, Oct 1983.

15 TROMBELLI, L.; SCHINCAGLIA, G. P.; ZANGARI, F.; SCAPOLI, C.; CALURA, G. Effect of pretreatment with ketorolac tromethamine on post-operative pain following periodontal surgery. J Clin Peridontol, v.23, n.2,p.128-132, Feb 1996.

16 VOGEL, R. I.; DESJARDINS, P. J.; MAJOR, K. V. O. Comparison of presurgical and immediate postsurgical ibuprofen on postoperative periodontal pain. J Periodontol, v.63, n.11, p.914-918, Nov 1992. 